\title{
Once Upon a Microscopic Slide: The Story of Histology
}

Inaya Hajj Hussein ${ }^{1^{*}}$, Mohamad Raad ${ }^{2}$, Rawan Safa ${ }^{2}$, Rosalyn Jurjus ${ }^{3}$ and Abdo Jurjus ${ }^{2}$

${ }^{1}$ Department of Biomedical Sciences, Oakland University William Beaumont School of Medicine, Rochester 48307, MI, USA

${ }^{2}$ Department of Anatomy, Cell biology and Physiology, Faculty of Medicine, American University of Beirut, Lebanon

${ }^{3}$ Department of Anatomy and Regenerative Biology, School of Medicine and Health Sciences, The George Washington University, Washington, D.C., United States.

"Corresponding author: Inaya Hajj Hussein, Department of Biomedical Sciences, OUWB School of Medicine, Rochester, MI 48309, USA, Tel: 2848217230; E-mail: HajjHuss@oakland.edu

Received date: Sep 29, 2015; Accepted date: Oct 17, 2015; Published date: Oct 19, 2015

Copyright: ( $\odot 2015$ Hussein $\mathrm{IH}$, et al. This is an open-access article distributed under the terms of the Creative Commons Attribution License, which permits unrestricted use, distribution, and reproduction in any medium, provided the original author and source are credited.

\begin{abstract}
For centuries, histology has maintained its remarkable place in the medical curriculum. However, its teaching has been influenced by the new technological advancement that has reshaped medicine teaching into a more modern student-friendly form. Since its inception in the 18th century, the discipline of histology has progressed hand in hand with the advancements in microscopy and microscopic technologies, including immunohistochemistry.

In the traditional curriculum of USA medical schools, especially after the first Flexner's report of 1910, histology was considered as very essential topic for a physician studying the "Art and Science" of medicine. In this era, the teaching relied more on the light microscope and to some extent on the electron microscope. However, the field nowadays, after the second Flexner's report, which stressed the importance of integrating clinical topics in the curriculum, is shifting towards the use of more electronic resources for teaching. Such new resources rely on information technology and electronic imaging modalities which are considered to be more student-friendly, time efficient, consistent in conveying the images, promote self-learning and are less costly. In fact, in the last 25 years, most universities started relying on virtual microscopy with limited use of the light microscopy by the students. Such an approach facilitated curricular integration of histology into histopathology and provided the opportunity to promote self-learning and clinical relevance. In the era of competency-based curriculum, histology remains an essential and indispensable basic science in the integrated modules.
\end{abstract}

Keywords: Histology; Microscopic anatomy; Virtual microscopy; Integrated modules

\section{Histology: A Fundamental Part of Medical Education}

Histology is the science that provides the adequate learning of the tissue organization of the body, and is a fundamental part of medical education [1]. Histology was traditionally taught as part of the basic science module during the first year of medical school. Its teaching has however been influenced by novel approaches to keep up with the trend of the new curricular changes. Histology is often considered to be the branch of anatomy that emphasizes the structural organization of tissues and provide together different perspectives to medicine [1]. However, if one digs deeper, histology is actually a separate discipline from Anatomy. The latter studies the gross physical structure of the body whereas the former goes into the molecular and cellular level of the body organs.

Teaching histology has been a challenge given the changes in the medical curriculum. Hence, it has adopted novel techniques and strategies to more efficiently and smoothly blend with the newer curriculum. Notably, the American medical schools have witnessed a decline in the total laboratory teaching time that medical students receive [2]. The lecture time in regards to histology has been minimized in order to keep the students' active attention. Moreover, the tools used in the laboratories have been dramatically changed from basic microscopy to computer-based techniques such as virtual slides, or an integration of both [3].

\section{The Father of Histology}

Histology, the study of details of tissues, came into usage in the 1700s by the scientist Marie François Xavier Bichat. Bichat is now considered to be the father of modern histology and descriptive anatomy. Bichat's work on 21 tissues was all based on gross dissection rather than the usage of the microscope [4]. Prior to that, Marcello Malpighi was the first scientist to observe capillaries, and thus was considered to be the true "Father of Histology" [5].

It was only until 1819 that Mayer coined the term "Histology". He combined two Greek root words that are histos, for tissues, and logos, for study. Histos originally was to describe any woven material, until Sir Richard Owen suggested the large usage of it in 1844 [5].

\section{The Evolution of the Microscope and Staining Techniques}

Microscopy has experienced great advancement since its creation. At the end of the 16th century, Jannsen, a Dutch eyeglass dealer, inserted lenses into a cylinder. He found out then that the objects were magnified, and that was the first prototype of modern microscopy. Jannsen later assembled the first microscope, which included two convex lenses [3]. In 1670, Dutch scientist Anthony van Leeuwenhoek evolved the method of the microscope of a higher magnification and better image quality. At that time, lenses were formed from beads and natural light; otherwise, light from a candle flame was needed to light the tissues [3]. 
During the 19th century, Ernst Abbe, Carl Zeiss, and Otto Schott worked on creating a high-quality microscope creating a foundation of discoveries for scientists in histology and anatomy [6]. The spectrum advanced from starting with a one-lens simple microscope to a two convex lenses microscope, to a virtual microscope and to what nowadays is known to be a 3-D microscope of moving cells [3].

Moreover, the use of paraffin wax for infiltration was introduced in the 19th century followed by formalin that came into use in 1893. The early staining techniques used by histologists and pathologists were derived from techniques developed by seventeenth century amateur scientists such as Leeuwenhoek. The 19th and 20th centuries were productive in terms of the new staining techniques in histology and histopathology. There were many different histology stains, and selection was according to the type of tissue to be observed. One of the oldest stains was Prussian blue, introduced in 1774. Furthermore, Perl's reaction, discovered in 1867 , is still widely used to localize intracellular iron and uses just Prussian blue for the histochemical localization of hemosiderin in tissues. Moreover, Wissowzky first described the hemaxtoxylin and eosin ( $\mathrm{H} \& \mathrm{E}$ stain) staining techniques in 1875-1878. Hematoxylin and eosinis the most commonly used histology stain for light microscopy.

As for Paul Ehrlich, Nobel Prize recipient in 1908, he worked on "magic bullets" and the use of dyes in order to discover and fight diseases. He developed the use of methylene blue in fighting malaria and trypan red for trypanosomes. Nowadays, there are many specific stains for the different kind of tissues [7]. These staining methods are generally inexpensive, reliable, fast, produce permanent preparations that are easy to interpret and archive, and deliver means for diagnoses that often cannot be achieved by other means. For these reasons, the different stains remain useful in the teaching of histology. Histology and histopathology have witnessed great changes in the past three decades, providing multiple means of reliable and fast methods of interpretations.

\section{Histology and Medicine: When did they Meet?}

"One must understand the normal to be able to recognize the abnormal" is a common phrase heard by medical students. Histology is the study of the tissues' anatomy, structure, and role. It is the study of tissues that make up the body organs, which keep the body functioning. It sheds light on the importance of the histological perspective in the study of body functions and the diagnosis of certain diseases. For example, in a study in the Journal of Clinical Oncology, on the prognostic significance of Nottingham Histologic Grade in Invasive Breast Carcinoma, the study proved that histological grading provides a strong predictor of outcome in patients that suffer from invasive carcinoma [8]. That also applies for several other cancers such as thyroid, lung, and colon cancer $[9,10]$.

Johannes Muller, a German scientist, was the first person to believe that microscopy can help the medical profession. He wrote the first book on the specialization of histopathology techniques entitled "On the Nature and Structural Characteristics of Cancer" in 1838. He elaborated that microscopy can prove a unique sense of investigation to different disease states. He believed it is the best way to track back diseases to their cellular and molecular malfunction. Muller's view was the foundation of the integration of histology into medicine. Moreover, it was then when histology became an integral part of the medical curriculum [1].
Ramon Y Cajal and Camillo Golgi both shared the Nobel Prize in Physiology and Medicine from their work on the nervous system structure [11]. Golgi was able to make great contributions to the understanding of the nervous system and its structure by creating a new histological method. In 1886, Cajal and a famous psychiatrist, professor Luis Simarro, discovered the histological method that was able to stain the entire neuron, which eventually helped in a better study of the neuron, and thus the nervous system.

The study of histology is essential for medical students in multiple ways. It helps students understand the arrangement of cells and tissues in a normal organ system. Moreover, it correlates the structure to function by correlating the differentiation of tissue structure to their specific function. This knowledge that students gain through histology will provide a better cellular understanding and basis of other important topics like anatomy and physiology. Most importantly, as previously stated, one must understand the normal to comprehend the abnormal. Thus, this makes Histology a fundamental aspect of pathology.

\section{Histology in the Traditional Curriculum}

More than a century ago, Abraham Flexner, a research scholar at the Carnegie Foundation for the Advancement of Teaching, visited around 155 medical schools in the United States to assess the form of education. He wrote a report in 1910 emphasizing the importance of scientific basis in teaching medicine and the importance of the scientific approach to preparing students for the medical profession. His views are still integrated in the medical curriculum but with changes on the methods they are integrated as.

Histology was initially given as a plain basic science course during the first year in medical school. It previously consisted of lectures followed by scoping glass slides in the laboratory. However, the field nowadays is shifting towards more electronic resources of teaching. Electronic resources are thought to be more student-friendly, more time efficient, and less costly [12]. Most universities started relying on virtual microscopy, which would use a single slide of the specimen, which is scanned and projected onto a screen where all students can view the image. It has become a widely used technology in the medical education of pathology and histology [12]. During the past 3 decades, various teaching strategies and activities were adopted to reach a good integration and offer anatomical sciences in a very clinically relevant approach that facilitates learning and promotes self-learning and critical thinking.

\section{Histology in the New Curriculum}

Over the years, the medical curriculum has undergone multiple improvements and changes. The basic sciences module seemed to fall behind at first but the new methods in teaching has helped it catch up. The new medical curriculum required teaching to move into a more digital imaging form. Improvements have been made in order to move from a passive highly detailed teaching of these studies to a more functional and clinically relevant method [13]. These improvements facilitated the changes schools were trying to implement. This curriculum tried to tackle self-learning amongst the students. The new curriculum has mostly tried to work on enhancing the students' work in the setting of the clinical cases. Even during the studying of basic sciences, there would be a period of self-directed studying and smallgroup discussions in order to stimulate the students' learning and application abilities [14]. Therefore, universities worked on reducing 
the student-faculty hours in order to provide the students with more time home to learn the material in the way they feel is most comfortable, and afterwards challenge themselves in-group discussions. Thus, this reduction would decompress the curriculum of the basic sciences that were given in too many details before [15]. In fact, most medical schools described their new curriculum as an active learning method. $57 \%$ of schools reported using problem-based learning and small-group discussion converging numerous branches of science in support of a new theory of learning [14]. Applying histology in real clinical cases early on falls in the new learning methods and helps the student in creating a big relevant picture based on existing histological knowledge.

In order to better implement the strategies of integration, the informatics tools required improvement as well [16]. In the university Universidad de Santander in Colombia, the newer teaching method of histology compared to the traditional methodology. The newer method integrates clinical medicine with basic science by combining clinical problems, gross pathology, histopathology, and autopsy pathology, all given through informatics tools. The traditional medical curriculum used in Western universities teaches pathology as an isolated subject in the preclinical years, and basic science concepts are imparted with limited clinical correlation. The study assessed the scores of 94 medical students for two consecutive semesters. The students were randomized based on the teaching they receive; either in the traditional methodology or through the new approach. The scores in the new approach group were significantly higher $3.91 / 5.0$ vs. $3.33 / 5.0, \mathrm{P}=$ 0.0008 . This emphasizes the potential virtual microscopy as well as digital imaging, carry in student learning [16].

How would these resources help students? In the system of the new curriculum, histology is put in parallel with pathology; thus, it provides a form of clinical aspect to learning. Moreover, some universities have a case-based format along with the histology images. Therefore, students would be able to attribute the images they see with a real life case scenario. Different universities have different viewpoints on why shifting from the light microscope laboratories and lecture time was an improvement. At Queen's University in Kingston in Canada, there is collaboration between the Department of Anatomy and Cell Biology, and the Department of Pathology and Molecular Medicine. There would be a group of clinical cases that include the chief complaint, past medical history, physical examination findings, lab tests, and imaging. Students are asked to understand all different angles to the story which includes the need for a biopsy, for example, to make the diagnosis. The biopsy findings will then be used to teach the histology material assigned to that session. This allows the creation of a connection between the basic sciences preclinical years and the clinical years. It allows students to understand the basic sciences, but in a more relevant clinical formulation, creating the bigger picture the new curriculum works on forming [17]. At Indiana University School of Medicine had adopted that change and wanted to test its effect on students' performance and the efficiency of the electronic and virtual microscopy teaching. Indiana University School of Medicine conducted a survey, which was done on individual test scores overall laboratory averages to determine the effects of virtual microscopy instead of the optical during human anatomy courses. A t-test analysis showed that the first two laboratory exams and the overall lab averages were significantly increased compared to years relying on optical microscopes. Students showed a preference to the electronic resources and lecture videos in the survey pointing out that increased access, ease of use, and their increased chances to understand the material made them choose virtual microscope teaching [18]. These studies come to prove that crossing paths and integrating different aspects of medical education is more efficacious in terms of students' commitments, interest, and scores.

In the University of Buffalo, curriculum changes were made in 2001, and the study of histology in School of Medicine was reformed. The time spent in histology classes was halved as virtual slides have replaced the traditional microscope. However, this has not reduced the amount of valuable information offered to students. This sheds light on how tedious and time demanding the traditional system of light microscopy was, a system that delivered the same information but in a more demanding method [15].

As for the New Jersey Medical School, They equipped histology labs with "Audiovisual Switching and Projection System", and thus projected images from glass slides, textbooks, videotapes, and 2'x2" slides. They also switched from Lecture-Laboratory-Review to Laboratory-LectureConference and topics start with a pre-lab presentation that helps students understand how to read the slides. Afterwards, students work on their own, implementing self-learning. Moreover, the lecture timing has been reduced greatly and is more oriented to the clinical training. Thus, the lecture time is no longer to describe histology and its details, but rather to correlate the structure to function, providing students an adequate understanding of the material. New Jersey Medical School had not implemented the use of virtual microscopy, but will probably have it implemented after it is "well-refined" [19].

The School of Michigan justifies its transition from the light microscopy with multiple reasons. First of, light microscopy usage in histology labs required a large number of glass slides and the maintenance of a great amount of microscopes which seemed to be a financial drain, which is a reason for most schools too. Moreover, since the histology glass slides are mostly of human origins, they are not only difficult to find but also difficult to be identical. Thus, there seemed to be some discrepancy between students and their slides. Allowing the students to learn the usage of the microscopy and its variability did not outweigh the financial drain that these institutions were facing [2].

As previously mentioned, virtual microscopy is the new tool used for histology teaching. Histological glass slides are picked based on the quality of preservation and staining. Then, the slides are scanned and converted into digital images [12]. These images are then transferred to special software or simply viewed as images which allow the students to pick their own region of interest from the slide and focus on it. Thus, all students receive an equal quality of slides, one that has a clearer view than the actual glass slides. An example of a software is ATLASplus [Advanced Tools for Learning Anatomical Structure], the multimedia program used to assist in the teaching of anatomy, embryology and histology in University of Michigan Medical School [20].

However, virtual microscopy, as with any other method, does not come without any drawbacks. First, there is loss of the 3dimensionality, where students move from different focus planes by changing their focus knobs. Students lose the sense of dimensions in regards to the slides they are viewing. Moreover, these software used do not only require technological structures at the institution, but also the ability to provide a big number of access to all students at once [21]. In 2006, the University of Michigan began using the virtual microscopy as the main tool of teaching, but kept light microscopy available upon request by the students. Over the last 6 years, less than 10 out of over 1,000 students took advantage of that offer [21]. As an alternative, the university decided to set up a number of microscopes 
with selected glass slides as a demonstration in the labs. Very few students used that opportunity to compare the virtual images to those of the real microscope. Actually, only 4 out of 142 students reported that they checked the demonstration microscopes every lab session. On the other hand, 92 students reported that they never used the demonstration microscopes during the entire histology course [2].

The use of virtual microscopy is now an accepted and often integral part of teaching histology [12]. Compared to the traditional methods, it helps medical schools teach the same amount of material given the cut down the basic sciences modules have faced in the newer curriculum. Moreover, it also allows better flexibility with student schedules and timing preference. However, it is definitely a costly approach. Will all schools and hospitals be able to move into the technological path, the one that uses sophisticated procedures of teaching?

On another note, many schools are now adopting what is known to be as the competency based curriculum. This curriculum focuses on learning more concrete rather than abstract skills [22]. It is like a form of assessment, grading, and reporting based on what students demonstrate of their knowledge and learning. Histology is providing case-based learning along with the images [23]. Moreover, from the first year, it divides the year into two semesters. The first year focuses on the basic sciences that are important and include histology. The other semester begins dividing the systems and with each, physiology, anatomy, histology, microbiology, genetics and pathology are included. However, even when taking basic sciences, the details that are not required for the clinical years are discarded [22].

According to the university of Michigan, technology and improvements are slowing down. New methods of teaching the microscopic sciences include software on Androids and iPads. Michigan University recently published "Michigan eHistology" as an application of self-evaluation on iPads which has a high image quality and great ease of use.

\section{What does the Future Hold for the Discipline of Histology?}

Even with the many changes that have hit the medical curriculum causing a decrease in focus on the basic sciences, histology remains an indispensable science to be taught. While it may not be given as a separate course by itself, histology is still being taught in integrated modules.

Histology has provided key information about biological tissues, animal growth, physiology, tissue diseases, and will continue to provide exciting findings in clinical medicine and research. Histopathology, its newer version examines the normal cellular structure of tissues and look for abnormalities. The ability to identify tissue abnormalities is a critical diagnostic tool and an irreplaceable field for the correct interpretation of clinical cases, their diagnosis, and prognosis [24].

\section{References}

1. Bennett HS (1956) The Role of Histology in Medical Education and Biological Thinking. Anat Rec 125: 327-354.

2. Bloodgood RA, Ogilvie RW (2006) Trends in histology laboratory teaching in United States medical schools. Anat Rec B New Anat 289: 169-175.
3. Chen X, Zheng B, Liu H (2011) Optical and digital microscopic imaging techniques and applications in pathology. Anal Cell Pathol (Amst) 34: 5-18.

4. Shoja MM, Tubbs RS, Loukas M, Shokouhi G, Ardalan MR (2008) MarieFrançois Xavier Bichat (1771-1802) and his contributions to the foundations of pathological anatomy and modern medicine. Ann Anat 190: 413-420.

5. http://www.mhhe.com/biosci/pae/zoology/hickman11/student/olc/ timeline.mhtml.

6. Davidson MW (2009) Ernest Abby Microscopy. LabMedicine 40: 502-503.

7. Bosch F, Rosich L (2008) The contributions of Paul Ehrlich to pharmacology: a tribute on the occasion of the centenary of his Nobel Prize. Pharmacology 82: 171-179.

8. Rakha EA, El-Sayed ME, Lee AH, Elston CW, Grainge MJ, et al. (2008) Prognostic significance of Nottingham histologic grade in invasive breast carcinoma. J Clin Oncol 26: 3153-3158.

9. Lo CY, Chan WF, Lam KY, Wan KY (2005) Follicular thyroid carcinoma: the role of histology and staging systems in predicting survival. Ann Surg 242: 708-715.

10. Aschebrook-Kilfoy B, Ward MH, Sabra MM, Devesa SS (2011) Thyroid cancer incidence patterns in the United States by histologic type, 1992-2006. Thyroid 21: 125-134.

11. De Carlos JA, Borrell J (2007) A historical reflection of the contributions of Cajal and Golgi to the foundations of neuroscience. Brain Res Rev 55: 8-16.

12. Donnelly AD, Mukherjee MS, Lyden ER, Radio SJ (2012) Virtual microscopy in cytotechnology education: Application of knowledge from virtual to glass. Cytojournal 9: 12.

13. Turney BW (2007) Anatomy in a modern medical curriculum. Ann R Coll Surg Engl 89: 104-107.

14. Irby DM, Wilkerson L (2003) Educational innovations in academic medicine and environmental trends. J Gen Intern Med 18: 370-376.

15. Cotter JR (2001) Laboratory instruction in histology at the University at Buffalo: recent replacement of microscope exercises with computer applications. Anat Rec 265: 212-221.

16. Diaz-Perez JA, Raju S, Echeverri JH (2014) Evaluation of a teaching strategy based on integration of clinical subjects, virtual autopsy, pathology museum, and digital microscopy for medical students. J Pathol Inform $5: 25$.

17. Hamilton PW, Wang Y, McCullough SJ (2012) Virtual microscopy and digital pathology in training and education. APMIS 120: 305-315.

18. Husmann PR, O'Loughlin VD, Braun MW (2009) Quantitative and qualitative changes in teaching histology by means of virtual microscopy in an introductory course in human anatomy. Anat Sci Educ 2: 218-226.

19. Haumont S, Cordier A, Dambrain R, Herveg JP, Rousseau P, et al. (1974) A new approach to teaching histology at the University of Louvain. Med Biol Illus 24: 202-206.

20. Chapman CM, Miller JG, Bush LC, Bruenger JA, Wysor WJ, et al. (1992) ATLAS-plus: multimedia instruction in embryology, gross anatomy, and histology. Proc Annu Symp Comput Appl Med Care.

21. Hortsch M (2013) Virtual biology: teaching histology in the age of Facebook. FASEB J 27: 411-413.

22. McGaghie WC, Miller GE, Sajid AW, Telder TV (1978) Competencybased curriculum development on medical education: an introduction. Public Health Pap 11-91.

23. Flåøyen A, Smith BL (1992) Parenchymal injury and biliary obstruction in relation to photosensitization in sporidesmin-intoxicated lambs. Vet Res Commun 16: 337-344.

24. Musumeci G (2014) Past, present and future: overview on histology and histopathology. Journal of Histology \& Histopathology 1: 5. 